OPEN ACCESS

Edited by:

Arun K. Bhunia,

Purdue University, United States

Reviewed by:

Roger Stephan,

University of Zurich, Switzerland

Bruce Michael Applegate,

Purdue University, United States

*Correspondence:

Renáta Karpiskova

karpiskova@vri.cz

Specialty section:

This article was submitted to

Food Microbiology,

a section of the journal

Frontiers in Microbiology

Received: 28 August 2020

Accepted: 09 December 2020

Published: 14 January 2021

Citation:

Gelbicova T, Florianova M, Hluchanova L, Kalova A, Korena K, Strakova N and Karpiskova R (2021)

Comparative Analysis of Genetic

Determinants Encoding Cadmium, Arsenic, and Benzalkonium Chloride Resistance in Listeria monocytogenes of Human, Food, and Environmental Origin. Front. Microbiol. 11:599882. do: 10.3389/fmicb.2020.599882

\section{Comparative Analysis of Genetic Determinants Encoding Cadmium, Arsenic, and Benzalkonium Chloride Resistance in Listeria monocytogenes of Human, Food, and Environmental Origin}

\author{
Tereza Gelbicova' ${ }^{1}$ Martina Florianova'1, Lucie Hluchanova',2, Alžběta Kalova'1, \\ Kristýna Korena ${ }^{1}$, Nicol Strakova ${ }^{1}$ and Renáta Karpiskova ${ }^{1,2 *}$
}

'Department of Microbiology and Antibiotic Resistance, Veterinary Research Institute, Brno, Czechia, ${ }^{2}$ Faculty of Veterinary Hygiene and Ecology, University of Veterinary and Pharmaceutical Sciences Brno, Brno, Czechia

Environmental adaptation of Listeria monocytogenes is a complex process involving various mechanisms that can contribute to their survival in the environment, further spreading throughout the food chain and the development of listeriosis. The aim of this study was to analyze whole-genome sequencing data in a set of 270 strains of L. monocytogenes derived from human listeriosis cases and food and environmental sources in order to compare the prevalence and type of genetic determinants encoding cadmium, arsenic, and benzalkonium chloride resistance. Most of the detected genes of cadmium (27.8\%), arsenic (15.6\%), and benzalkonium chloride (7.0\%) resistance were located on mobile genetic elements, even in phylogenetically distant lineages I and II, which indicates the possibility of their horizontal spread. Although no differences were found in the prevalence of these genes between human and food strains, they have been detected sporadically in strains from the environment. Regarding cadmium resistance genes, cadA1C1_Tn5422 predominated, especially in clonal complexes (CCs) 121, 8, and 3 strains. At the same time, qacH_Tn6188-encoding benzalkonium chloride resistance was most frequently detected in the genome of CC121 strains. Genes encoding arsenic resistance were detected mainly in strains CC2 (located on the chromosomal island LGI2) and CC9 (carried on Tn554). The results indicated a relationship between the spread of genes encoding resistance to cadmium, arsenic, and benzalkonium chloride in certain serotypes and CCs and showed the need for a more extensive study of $L$. monocytogenes strains to better understand their ability to adapt to the food production environment.

Keywords: Listeria, comparative genomics, cadmium, arsenic, benzalkonium chloride, resistance, mobile genetic elements 


\section{INTRODUCTION}

Listeria monocytogenes is a facultative intracellular pathogen and at the same time ubiquitous saprophytic bacterium, which can also be isolated from the soil, water, and vegetation (Gray et al., 2006). L. monocytogenes is also known for its ability to persist in the food-processing environment. However, unique properties that would be entirely responsible for the persistence of Listeria have not yet been revealed (Carpentier and Cerf, 2011). L. monocytogenes can be considered a genetically heterogeneous species. Its isolates have been classified into a number of clonal complexes (CCs) with specific characteristics and frequency of occurrence. At present, the classification of L. monocytogenes into hypervirulent and hypovirulent clones is also used. The hypervirulent clones include strains of CC1, CC2, CC4, and CC6, which are mainly associated with human and ruminant listeriosis cases, and the hypovirulent clones, typically containing truncated internalin A, include strains of CC121 and CC9, which are mainly associated with the occurrence and persistence in food-processing environments (Maury et al., 2019).

The cadmium and arsenic resistance encoded by different genetic determinants often located on mobile genetic elements was one of the earliest environmental adaptations observed in L. monocytogenes (Parsons et al., 2019). The mechanisms of cadmium resistance known thus far include efflux pumps encoded by the following genes: cadA1C1, which are commonly located on the plasmid-carried transposon Tn5422; cadA2C2 first described on plasmid pLM80; cadA3C3 carried on the integrative and conjugative element (ICE); and cadA4C4 located on Listeria Genomic Island 2 (LGI2) (Parsons et al., 2020). Even though cadmium resistance in Listeria can often be spread by horizontal gene transfer, arsenic resistance in L. monocytogenes is encoded mainly chromosomally by the $\operatorname{arsR} 1 D 2 R 2 A 2 B 1 B 2$ cassette carried together with cadA4C4 on LGI2 (Kuenne et al., 2013; Parsons et al., 2019). The insertion of mobile element LGI2 into the chromosome is likely mediated by phage integrase (Kuenne et al., 2013). The presence of this cassette of arsenic resistance genes on plasmids has been found occasionally in L. monocytogenes (Hingston et al., 2019). The arsCBADR operon located on Tn554 transposon may also be involved in arsenic resistance in L. monocytogenes (Kuenne et al., 2013; Lee et al., 2017).

Arsenic resistance encoded by genes carried on LGI2 is primarily associated with its occurrence in L. monocytogenes strains of $4 \mathrm{~b}$ serotype, especially in the hypervirulent clones CC1, CC2, and CC4 (Lee et al., 2017). Conversely, in strains of serotypes $1 / 2 \mathrm{a}, 1 / 2 \mathrm{~b}$, and $1 / 2 \mathrm{c}$, the presence of LGI2 is rarely reported (Lee et al., 2017; Hingston et al., 2019). However, underlying mechanism of the effect of arsenic resistance genes or other genes of genomic island LGI2 on L. monocytogenes virulence remains to be elucidated. The only described mechanism demonstrating the effect of genes encoding heavy metal resistance on L. monocytogenes virulence is the cadC3 gene, which, in addition to $c a d A 3$ regulation, suppresses $l s p B$ gene expression and thus promotes Listeria survival in macrophages (Pombinho et al., 2017).
Conversely, cadmium resistance is more commonly described in L. monocytogenes strains of serotypes $1 / 2 \mathrm{a}$ and $1 / 2 \mathrm{c}$ isolated from food and food-processing environment (Mullapudi et al., 2008; Ratani et al., 2012), although Listeria is not exposed to any obvious selection pressure related to cadmium-related exposure in food-processing plants. In Northern Ireland, cadmium resistance was detected more frequently in repeatedly isolated L. monocytogenes from milk and non-dairy products than in sporadic strains obtained from eight different producers (Harvey and Gilmour, 2001). Similarly, the occurrence of different variants of resistance genes not only to cadmium, but also to arsenic, has been reported in persistent strains of L. monocytogenes CC14 and CC121 isolated from a rabbit meatprocessing plant in Italy (Pasquali et al., 2018). The possible effect of these genes on the persistence of $L$. monocytogenes in food-processing plants has not yet been confirmed.

The resistance of $L$. monocytogenes to cadmium often correlates with the resistance to quaternary ammonium compounds, such as benzalkonium chloride, widely used for disinfection in the food industry (Mullapudi et al., 2008; Ratani et al., 2012). The benzalkonium chloride resistance is one of the factors that could contribute to the persistence of Listeria in food-processing facilities. The benzalkonium chloride resistance in $L$. monocytogenes is mediated by the activity of various efflux pumps encoded by the $b c r A B C$ cassette carried on plasmid pLM80 (Elhanafi et al., 2010), by qacH gene chromosomally located on the Tn6188 transposon (Müller et al., 2013), qacA and qacC genes carried by plasmids (Xu et al., 2014; Hurley et al., 2019), emrE gene located chromosomally on LGI1 (Kovacevic et al., 2015), and emrC first described on plasmid pLMST6 (Kremer et al., 2017). In addition to efflux pumps carried on mobile genetic elements, the tolerance of L. monocytogenes to benzalkonium chloride can also be affected by the chromosomal multidrug efflux pump MdrL (Jiang et al., 2019). The plasmid-carried $b c r A B C$ cassette and the cadA1 and cadA2 genes are also found in non-pathogenic Listeria species (e.g., Listeria innocua, Listeria welshimeri) commonly detected in food-processing plants, which can become sources of these genes for L. monocytogenes (Katharios-Lanwermeyer et al., 2012; Korsak et al., 2019).

Even though the environment is burdened by anthropogenic activities (industry, agriculture), information on the occurrence of genetic determinants encoding resistance to heavy metals in L. monocytogenes strains isolated from environmental samples is missing up to now. Heavy metals are found in disinfectants, soil fertilizers, and livestock feeding and are recognized as environmental pollutants. Therefore, bacterial populations, with genetic determinants conferring resistance to heavy metals, can be found in the gut microbiota of intensively reared farm animal species. The pollution of soils with heavy metals is variable, virtually inextricable, and can contribute to the contamination of water sources (Doležalová Weissmannová and Pavlovský, 2017). At present, the differences between the occurrence of resistance genes to heavy metals such as arsenic and cadmium or quaternary ammonium compounds in L. monocytogenes clones isolated from various sources (humans, food, and environment) are not fully understood. In the Czechia, to date no study focused on 
the occurrence of genetic determinants encoding resistance to heavy metals and disinfectants in L. monocytogenes has yet been performed. Therefore, the aim of the present study was to carry out a comparative analysis of the occurrence and type of genetic determinants encoding cadmium, arsenic, and benzalkonium chloride resistance in L. monocytogenes from various sources, focusing on their clonal determination and origin of isolation.

\section{MATERIALS AND METHODS}

\section{Tested Strains of $L$. monocytogenes}

A total of 270 L. monocytogenes strains isolated from food $(n=106)$, mainly ready-to-eat food $(n=92)$, including strains from meat $(n=45)$, dairy $(n=23)$, and fish $(n=6)$ products; delicatessen $(n=12)$; fresh vegetables $(n=6)$; raw meat $(n=6)$; and frozen vegetables $(n=8)$, from clinical cases of human listeriosis $(n=132)$ and the environment $(n=32)$ were collected between 2010 and 2020. Strains from the environment were obtained mainly from mud on the banks of surface water sources $(n=17)$, rotting vegetation $(n=5)$, soil $(n=5)$, surface water $(n=2)$, algae $(n=1)$, and moss $(n=2)$. Only one strain per each human listeriosis outbreak was included in the analysis, food strains originated from different producers. The strains were selected to reflect the proportions of L. monocytogenes CCs in humans and in food in the Czechia (Tomáštíková et al., 2019). All available environmental strains stored in the cryobank at the VRI in Brno, classified into serogroups by the matrix-assisted laser desorption ionization-time of flight mass spectrometry method, were analyzed (Koudelka et al., 2018). Strains of serotype 1/2a $(n=136)$ belonged to 24 CCs and 31 STs: CC7 $(n=7)$, CC8 $(n=31), \operatorname{CC} 11(n=1), \operatorname{CC} 14(n=5), \operatorname{CC} 18(n=4), \operatorname{CC} 19$ $(n=2), \operatorname{CC} 20(n=5), \operatorname{CC} 21(n=5), \operatorname{CC} 26(n=3), \operatorname{CC} 29(n=3)$, CC31 $(n=1), \operatorname{CC} 37(n=9), \operatorname{CC101}(n=4), \operatorname{CC} 121(n=14)$, CC124 $(n=2), \operatorname{CC155}(n=8), \operatorname{CC} 193(n=1), \operatorname{CC} 200(n=1)$, CC204 $(n=6), \operatorname{CC} 398(n=1), \operatorname{CC} 403(n=3), \operatorname{CC} 415(n=1)$, CC451 $(n=16)$, and CC475 $(n=3)$. Strains of serotype $1 / 2 \mathrm{~b}$ $(n=40)$ belonged to five CCs and nine STs: CC3 $(n=13)$, CC5 $(n=7), \operatorname{CC} 87(n=18), \operatorname{CC} 195(n=1)$, and CC288 $(n=1)$. Strains of serotype $4 \mathrm{~b}(n=79)$ belonged to five CCs and nine STs: CC1 $(n=27)$, CC2 $(n=21)$, CC4 $(n=3)$, CC6 $(n=26)$, and CC315 $(n=2)$. Strains of serotype $1 / 2 c(n=15)$ belonged to CC9. Detailed information about CCs and STs of strains isolated from various sources is mentioned in the Supplementary Table.

\section{Whole-Genome Sequencing}

Genomic DNA was extracted using the Blood and Tissue kit according to the manufacturer's instructions (Qiagen, Germany). Preparation of DNA libraries by Nextera XT DNA Library Preparation Kit (Illumina) and sequencing on the Illumina platform were carried out externally using MiSeq $(n=154)$ and NextSeq $(n=116)$ sequencers.

\section{Whole-Genome Sequencing Data Quality Assessment}

Quality of the reads was checked for all fastq files using FastQC in Unipro UGENE v. 36 (Okonechnikov et al., 2012). Phred score greater than 30 was set as short-reads set quality criteria. Average depth coverage greater than $30 \times$ was required. The genome size (between 2.8 and $3.1 \mathrm{Mb}$ ), consistent with L. monocytogenes parameters, was considered as assembly quality criteria.

\section{Genome Analysis}

The raw sequence data were assembled using the Velvet assembler version 1.1.04 integrated in Ridom SeqSphere ${ }^{+}$ software (version 6.0.2; Ridom GmbH, Münster, Germany) using optimized k-mer size and coverage cutoff values based on the average length of contigs with $>1000 \mathrm{bp}$; reads were trimmed at their 5 ' and 3' ends until an average base quality of 30 was reached in a window of 20 bases. The nucleotide sequences of selected benzalkonium chloride, cadmium, and arsenic resistance genes were downloaded from the NCBI database (Supplementary Table). The presence of the monitored genes was detected using Ridom SeqSphere ${ }^{+}$software. The analysis was performed at default setting, i.e., reference sequence identity was at least $90 \%$, and base sequence identity with reference sequence was $99 \%$. The presence of plasmids was searched for with the PlasmidFinder v2.1 tool (Carattoli et al., 2014) at https://cge.cbs.dtu.dk/services/. The sequences of benzalkonium chloride, cadmium, and arsenic resistance genes were annotated by Prokka 1.14.5 software. BLAST (The Basic Local Alignment Search Tool) available at https://blast.ncbi.nlm.nih.gov/Blast.cgi was used to verify the presence of plasmids pLM80 (GenBank AADR01000010) and pLMST6 (GenBank LT732640), the presence of transposons Tn6188 (GenBank HG329628), Tn5422 (GenBank L28104) and Tn554 (GenBank FR33648), and also the presence of the complete LGI2 sequence (GenBank CM001159) in the tested strains in which benzalkonium chloride, cadmium, and arsenic resistance genes were detected using Ridom SeqSphere ${ }^{+}$and Prokka software.

\section{RESULTS}

\section{Distribution of Cadmium, Arsenic, and Benzalkonium Chloride Resistance Genes in L. monocytogenes From Various Sources}

In L. monocytogenes strains isolated from food, in sequences obtained by whole-genome sequencing, resistance genes to cadmium (36.8\%), arsenic (23.6\%), and benzalkonium chloride $(9.4 \%)$ were detected more frequently in comparison with strains isolated from human cases of listeriosis (Table 1). In strains isolated from the environment, cadmium, arsenic, and benzalkonium chloride resistance genes were present only occasionally (Table 1 ). Out of the L. monocytogenes strains obtained from the environment, cadmium and arsenic resistance genes were detected in a strain isolated from decaying vegetation, whereas cadmium resistance genes and simultaneously benzalkonium chloride resistance genes were found in a strain isolated from pond water. In both cases, the strains originated from locations used for recreational activities outside industrial and agricultural areas. However, no significant 
TABLE 1 | Occurrence of genes encoding resistance to benzalkonium chloride, cadmium, and arsenic in $L$. monocytogenes of various origin.

\begin{tabular}{|c|c|c|c|c|}
\hline \multirow[t]{2}{*}{ Origin of strains } & \multirow[t]{2}{*}{ No. of tested strains } & \multicolumn{3}{|c|}{ No. of strains $(\%)$ carrying genes encoding resistance to } \\
\hline & & Benzalkonium chloride & Cadmium & Arsenic \\
\hline Human & 132 & $8(6.1 \%)$ & 35 (26.5\%) & $16(12.1 \%)$ \\
\hline Food & 106 & $10(9.4 \%)$ & $39(36.8 \%)$ & $25(23.6 \%)$ \\
\hline Environment & 32 & $1(3.1 \%)$ & $2(6.3 \%)$ & $1(3.1 \%)$ \\
\hline Total & 270 & 19 (7.0\%) & 75 (27.8\%) & 42 (15.6\%) \\
\hline
\end{tabular}

difference in the prevalence of these genes was observed between strains from food and human cases. The co-occurrence of genes encoding cadmium and benzalkonium chloride resistance was found in $13(4.8 \%)$ L. monocytogenes strains isolated from food $(n=6)$, human cases of listeriosis $(n=6)$, and the environment $(n=1)$. Arsenic resistance genes were detected mainly on the chromosomal island LGI2 together with cadmium resistance genes (in $10 \%$ of strains) in strains from human listeriosis $(n=15)$, food $(n=11)$, and environment $(n=11)$.

\section{Occurrence and Types of Genetic Determinants Encoding Cadmium, Arsenic, and Benzalkonium Chloride Resistance}

Genetic determinants of $L$. monocytogenes resistance to heavy metals, especially to cadmium (27.8\%), were more frequently detected in the tested strains than resistance genes to benzalkonium chloride (7.0\%). Arsenic resistance genes were detected in the genome of $15.6 \%$ strains. Regarding the genes encoding cadmium resistance, the cadA1C1 genes carried on the Tn5422 transposon predominated in the tested strains (17.8\%), followed by the cadA4C4 genes (10.0\%) located on the LGI2 chromosomal island together with the genes encoding arsenic resistance (Table 2). In most strains positive for the cadA1C1 (42/48) genes, Tn5422 was typically carried on plasmids. In two strains, isolated from a clinical case of listeriosis and from a meat product, belonging to $\mathrm{CC} 124, \mathrm{Tn} 5422$ was located on the chromosome. In four strains, the location of $\operatorname{Tn} 5422$ could not be found according to the obtained sequencing data (Supplementary Table). The cadA2C2 genes were detected in parallel with the $b c r A B C$ cassette on plasmids in $1.1 \%$ of the tested strains. The $c a d A 3 C 3$ genes were not detected in any of the tested L. monocytogenes strains. Regarding genetic determinants encoding arsenic resistance, the $\operatorname{ars} C B A D R$ operon located on the Tn554 transposon (5.6\%) was identified in the genome of the tested strains in addition to LGI2. The analysis of the obtained sequences revealed chromosomal location of the Tn554 transposon in the tested strains.

Benzalkonium chloride resistance genes were carried almost exclusively on mobile genetic elements and were detected in 7.0\% of the L. monocytogenes strains tested. Benzalkonium chloride resistance was most often encoded by the qacH gene located on the Tn6188 transposon (4.1\%). Four strains of L. monocytogenes isolated from humans $(n=3)$ and vegetables $(n=1)$ with the $q a c H$ gene located on the chromosome, which did not carry Tn6188 in the genome (Table 2), belonged to four different CCs
(Supplementary Table). In the qacH gene of these strains, an insertion of guanine at position 365 (365insG, Met124X) was detected in contrast to the reference sequence (Müller et al., 2013). Subsequently, by comparing with the qacH sequences available in the NCBI database, they were identified as $100 \%$ identical with qacH MK944277 of L. monocytogenes (NCBI, Blast). The occurrence of $b c r A B C$ genes (1.1\%) and the emrC gene $(0.4 \%)$ located on the plasmids was occasional. By comparing the obtained sequences, it was found that plasmids carrying the bcr $A B C$ cassette together with cadA2C2 belonged to the originally described plasmid type pLM80 (Elhanafi et al., 2010), and the emrC gene was located on the plasmid pLMST6 (Kremer et al., 2017). Neither the qacA and qacC genes located on plasmids nor the emrE gene located on chromosomal island LGI1 was detected in the tested strains (Table 2).

\section{Comparison of Cadmium, Arsenic, and Benzalkonium Chloride Resistance Genes in Different $L$. monocytogenes Clones}

The prevalence of cadmium resistance genes was comparable in strains from lineage I (serotype $4 \mathrm{~b}, 1 / 2 \mathrm{~b}$ ) and lineage II (serotypes $1 / 2 \mathrm{a}, 1 / 2 \mathrm{c}$ ), namely, 27.7 and $28.5 \%$. It was similar in arsenic resistance genes, where the prevalence found in strains of lineage I $(16.8 \%)$ was slightly higher compared to strains of lineage II (14.6\%). No arsenic resistance genes were detected in any tested strain of serotype $1 / 2 \mathrm{~b}$. In contrast, benzalkonium chloride resistance genes were more frequently detected in lineage II strains $(10.6 \%)$ compared to lineage I strains $(2.5 \%)$, regardless of the origin of the strains.

Out of the detected 35 CCs of L. monocytogenes, resistance genes to benzalkonium chloride and heavy metals were detected only in strains belonging to 17 of them: CC8, CC14, CC20, CC21, CC31, CC101, CC121, CC124, CC155, CC193, CC204, CC288 (serotype 1/2a), CC9 (serotype 1/2c), CC2 (serotype 4b), CC3, CC5, and CC195 (serotype 1/2b). The prevalence of genes in strains belonging to different CCs is shown in Table 2. The prevalence of genes encoding benzalkonium chloride, cadmium, and arsenic resistance was typical of certain CCs. However, these genes were not found in the genome of any of the tested strains of the given CC (Figures 1A,B). These were, for example, cadmium resistance genes cadA1C1, which occurred mainly in the genome of strains CC121 (13/14; 93\%), CC3 (11/13; 85\%), CC8 (11/31; $35 \%)$, and CC9 $(5 / 15 ; 33 \%)$. On the other hand, the genes of LGI2 chromosomal island encoding cadmium and arsenic resistance occurred mainly in CC2 strains (20/21; 95\%), in all tested 
TABLE 2 | Distribution of genes encoding resistance to benzalkonium chloride, cadmium, and arsenic in tested L. monocytogenes strains.

\begin{tabular}{|c|c|c|c|c|c|c|}
\hline Resistance to & Gene & Localization & $\begin{array}{c}\text { No. (\%) of positive } \\
\text { strains }\end{array}$ & $\begin{array}{l}\text { Origin of } \\
\text { strains }\end{array}$ & Serotype & $\begin{array}{l}\text { CCs (no. of positive } \\
\text { strains) }\end{array}$ \\
\hline \multirow[t]{6}{*}{$\begin{array}{l}\text { Benzalkonium } \\
\text { chloride }\end{array}$} & qacH & Transposon_Tn6188 & $11(4.1 \%)$ & $\begin{array}{l}\text { Human, food, } \\
\text { environment }\end{array}$ & $\begin{array}{l}1 / 2 a \\
1 / 2 c \\
4 b\end{array}$ & $\begin{array}{l}\text { CC121 (8); CC101 (1) } \\
\text { CC9 (1) } \\
\text { CC2 (1) }\end{array}$ \\
\hline & & Chromosome & $4(1.5 \%)$ & human, food & $\begin{array}{l}1 / 2 a \\
1 / 2 b\end{array}$ & $\begin{array}{l}\text { CC8 (1); CC20 (1); } \\
\text { CC121 (1) } \\
\text { CC3 (1) }\end{array}$ \\
\hline & qacA & Plasmid & ND & ND & ND & ND \\
\hline & qacC & Plasmid & ND & ND & ND & ND \\
\hline & emrE & Chromosome_LGl1 & ND & ND & ND & ND \\
\hline & emrC & Plasmid_pLMST6 & $1(0.4 \%)$ & Food & $1 / 2 a$ & CC8 (1) \\
\hline \multirow[t]{5}{*}{ Cadmium } & $\begin{array}{l}\text { bcrABC } \\
\text { cadA2C2 }\end{array}$ & Plasmid_pLM80 & $3(1.1 \%)$ & Human, food & $\begin{array}{l}1 / 2 a \\
1 / 2 b\end{array}$ & $\begin{array}{l}\text { CC204 (2) } \\
\text { CC288 (1) }\end{array}$ \\
\hline & $\operatorname{cadA1C1}$ & Transposon_Tn5422 & $48(17.8 \%)$ & $\begin{array}{l}\text { Human, food, } \\
\text { environment }\end{array}$ & $1 / 2 \mathrm{a}$ & $\begin{array}{l}\text { CC121 (13); CC8 } \\
\text { (11); CC124 (2); } \\
\text { CC21 (1); CC31 (1); } \\
\text { CC155 (1); CC193 (1) }\end{array}$ \\
\hline & & & & & $1 / 2 c$ & $\operatorname{cc9}(5)$ \\
\hline & & & & & $1 / 2 b$ & $\begin{array}{l}\text { CC3 (11); CC5 (1); } \\
\text { CC195 (1) }\end{array}$ \\
\hline & cadАЗСЗ & Chromosome & ND & ND & ND & ND \\
\hline \multirow[t]{2}{*}{ Arsenic } & $\begin{array}{l}\text { cadA4C4 } \\
\operatorname{ars} A 1 A 2 B 1 B 2 D 1 D 2 R 1 R 2\end{array}$ & Chromosome_LGI2 & 27 (10.0\%) & $\begin{array}{l}\text { Human, food, } \\
\text { environment }\end{array}$ & $\begin{array}{c}1 / 2 a \\
4 b\end{array}$ & $\begin{array}{l}\text { CC204 (6); CC14 (1) } \\
\text { CC2 (20) }\end{array}$ \\
\hline & $\operatorname{ars} C B A D R$ & Transposon_Tn554 & 15 (5.6\%) & Human, food & $1 / 2 c$ & CC9 (15) \\
\hline
\end{tabular}

ND, not detected; CCs, clonal complexes; LGl1, Listeria genetic island 1; LGI2, Listeria genetic island 2.

strains CC204 (6/6) and in one of the five tested strains CC14 (Figures 1A,B). The comparison of the detected chromosomal islands LGI2 in strain serotype 4b CC2 and serotype 1/2a CC204 and CC14 showed 100\% identity. In addition to LGI2, two strains in CC204 isolated from ready-to-eat foods (ripened cheese and vegetables) simultaneously carried the cadA2C2 and bcrABC genes located within plasmids. The resistance gene arsCBADR on Tn554 was detected only in serotype $1 / 2 \mathrm{c}$ strains in the genome of all tested CC9 strains, isolated mainly from readyto-eat meat products $(n=9)$, raw meat $(n=5)$, and also from human $(n=1)$.

\section{DISCUSSION}

\section{Distribution of Cadmium, Arsenic, and Benzalkonium Chloride Resistance Genes Among L. monocytogenes Strains From Humans, Food, and the Environment}

Given the foodborne origin of listeriosis, it is not surprising that there was no significant difference in the prevalence of the monitored resistance genes to heavy metals and benzalkonium chloride between human and food strains collected in the Czechia. Twenty-three of 35 CCs (66\%) tested in this study belonged to both strains of human and food origin. Environmental strains also belonged to the same CCs $(9 / 13,69 \%)$ detected (Supplementary Table). However, it was noteworthy that the tested strains isolated from the environment (e.g., soil, water), in which selection pressure could be expected due to anthropogenic activity, rarely carried genes of resistance to cadmium $(6.3 \%)$ and arsenic (3.1\%). One of the serotype $1 / 2 \mathrm{a}$ strains, CC204, isolated from decaying vegetation carried arsenic and cadmium resistance genes in LGI2. Another strain isolated from pond water belonged to CC121 and carried the resistance genes to cadmium cadA1C1 located on Tn5422 and at the same time the benzalkonium chloride resistance gene qacH on Tn6188. Both strains were isolated from sites without a direct industrial or agricultural burden. The rare detection of genetic determinants encoding heavy metal resistance in L. monocytogenes from the environment of the Czechia in this study may be due to the limited number of available strains tested in this group and the related low variability of CCs.

At present, there is a lack of studies to compare the occurrence of cadmium and arsenic resistance genes in L. monocytogenes in relationship to the source and country of origin. However, the prevalence of genes encoding resistance to cadmium 
A \begin{tabular}{|l|l|l|l|l|}
\hline 0 & $1-25 \%$ & $26-50 \%$ & $51-75 \%$ & $76-100 \%$ \\
\hline
\end{tabular}

\begin{tabular}{|c|c|c|c|c|c|c|c|c|c|c|c|c|}
\hline Origin & $q a c H$ & $q a c A$ & qacc & emrE & emrC & bcrABC & cadA2C2 & cadA1C1 & cadA3C3 & cadA4C4 & ars_LGI2 & $\operatorname{ars} C B A D R$ \\
\hline Human & $7 / 132$ & $0 / 132$ & $0 / 132$ & $0 / 132$ & $0 / 132$ & $1 / 132$ & $1 / 132$ & $19 / 132$ & $0 / 132$ & $15 / 132$ & $15 / 132$ & $1 / 132$ \\
\hline Food & $7 / 106$ & $0 / 106$ & $0 / 106$ & $0 / 106$ & $1 / 106$ & $2 / 106$ & $2 / 106$ & $28 / 106$ & $0 / 106$ & $11 / 106$ & $11 / 106$ & $14 / 106$ \\
\hline Environment & $1 / 32$ & $0 / 32$ & $0 / 32$ & $0 / 32$ & $0 / 32$ & $0 / 32$ & $0 / 32$ & $1 / 32$ & $0 / 32$ & $1 / 32$ & $1 / 32$ & $0 / 32$ \\
\hline Total & $15 / 270$ & $0 / 270$ & $0 / 270$ & $0 / 270$ & $1 / 270$ & $3 / 270$ & $3 / 270$ & $48 / 270$ & $0 / 270$ & $27 / 270$ & $27 / 270$ & $15 / 270$ \\
\hline
\end{tabular}

\begin{tabular}{|c|c|c|c|c|c|c|c|c|c|c|c|c|c|c|}
\hline \multirow[t]{5}{*}{ Lineage } & \multirow[t]{3}{*}{ Serotype } & CCs & qacH & $q a c A$ & qacc & emrE & emrC & $b c r A B C$ & cadA2C2 & cadA1C1 & cadA3C3 & cadA4C4 & ars_LGI2 & $\operatorname{ars} C B A D R$ \\
\hline & & CC1 & $0 / 27$ & $0 / 27$ & $0 / 27$ & $0 / 27$ & $0 / 27$ & $0 / 27$ & $0 / 27$ & $0 / 27$ & $0 / 27$ & $0 / 27$ & $0 / 27$ & $0 / 27$ \\
\hline & & CC2 & $1 / 21$ & $0 / 21$ & $0 / 21$ & $0 / 21$ & $0 / 21$ & $0 / 21$ & $0 / 21$ & $0 / 21$ & $0 / 21$ & $20 / 21$ & $20 / 21$ & $0 / 21$ \\
\hline & \multirow[t]{3}{*}{$4 b$} & CC4 & $0 / 3$ & $0 / 3$ & $0 / 3$ & $0 / 3$ & $0 / 3$ & $0 / 3$ & $0 / 3$ & $0 / 3$ & $0 / 3$ & $0 / 3$ & $0 / 3$ & $0 / 3$ \\
\hline & & CC6 & $0 / 26$ & $0 / 26$ & $0 / 26$ & $0 / 26$ & $0 / 26$ & $0 / 26$ & $0 / 26$ & $0 / 26$ & $0 / 26$ & $0 / 26$ & $0 / 26$ & $0 / 26$ \\
\hline \multirow{6}{*}{1} & & CC315 & $0 / 2$ & $0 / 2$ & $0 / 2$ & $0 / 2$ & $0 / 2$ & $0 / 2$ & $0 / 2$ & $0 / 2$ & $0 / 2$ & $0 / 2$ & $0 / 2$ & $0 / 2$ \\
\hline & \multirow{5}{*}{$1 / 2 b$} & CC3 & $1 / 13$ & $0 / 13$ & $0 / 13$ & $0 / 13$ & $0 / 13$ & $0 / 13$ & $0 / 13$ & $11 / 13$ & $0 / 13$ & $0 / 13$ & $0 / 13$ & $0 / 13$ \\
\hline & & CC5 & $0 / 7$ & $0 / 7$ & $0 / 7$ & $0 / 7$ & $0 / 7$ & $0 / 7$ & $0 / 7$ & $1 / 7$ & $0 / 7$ & $0 / 7$ & $0 / 7$ & $0 / 7$ \\
\hline & & CC87 & $0 / 18$ & $0 / 18$ & $0 / 18$ & $0 / 18$ & $0 / 18$ & $0 / 18$ & $0 / 18$ & $0 / 18$ & $0 / 18$ & $0 / 18$ & $0 / 18$ & $0 / 18$ \\
\hline & & CC195 & 0/1 & $0 / 1$ & 0/1 & $0 / 1$ & $0 / 1$ & $0 / 1$ & $0 / 1$ & $1 / 1$ & $0 / 1$ & $0 / 1$ & $0 / 1$ & $0 / 1$ \\
\hline & & CC288 & $0 / 1$ & $0 / 1$ & $0 / 1$ & $0 / 1$ & $0 / 1$ & $1 / 1$ & $1 / 1$ & $0 / 1$ & $0 / 1$ & $0 / 1$ & $0 / 1$ & $0 / 1$ \\
\hline \multirow{25}{*}{ II } & \multirow{24}{*}{$1 / 2 a$} & CC7 & $0 / 7$ & $0 / 7$ & $0 / 7$ & $0 / 7$ & $0 / 7$ & $0 / 7$ & $0 / 7$ & $0 / 7$ & $0 / 7$ & $0 / 7$ & $0 / 7$ & $0 / 7$ \\
\hline & & CC8 & $1 / 31$ & $0 / 31$ & $0 / 31$ & $0 / 31$ & $1 / 31$ & $0 / 31$ & 0/31 & $11 / 31$ & 0/31 & $0 / 31$ & $0 / 31$ & 0/31 \\
\hline & & CC11 & 0/1 & $0 / 1$ & 0/1 & 0/1 & $0 / 1$ & $0 / 1$ & $0 / 1$ & $0 / 1$ & $0 / 1$ & $0 / 1$ & $0 / 1$ & $0 / 1$ \\
\hline & & CC14 & $0 / 5$ & $0 / 5$ & $0 / 5$ & $0 / 5$ & $0 / 5$ & $0 / 5$ & $0 / 5$ & $0 / 5$ & $0 / 5$ & $1 / 5$ & $1 / 5$ & $0 / 5$ \\
\hline & & CC18 & $0 / 4$ & $0 / 4$ & $0 / 4$ & $0 / 4$ & $0 / 4$ & $0 / 4$ & $0 / 4$ & $0 / 4$ & $0 / 4$ & $0 / 4$ & $0 / 4$ & $0 / 4$ \\
\hline & & CC19 & $0 / 2$ & $0 / 2$ & $0 / 2$ & $0 / 2$ & $0 / 2$ & $0 / 2$ & $0 / 2$ & $0 / 2$ & $0 / 2$ & $0 / 2$ & $0 / 2$ & $0 / 2$ \\
\hline & & CC20 & $1 / 5$ & $0 / 5$ & $0 / 5$ & $0 / 5$ & $0 / 5$ & $0 / 5$ & $0 / 5$ & $0 / 5$ & $0 / 5$ & $0 / 5$ & $0 / 5$ & $0 / 5$ \\
\hline & & CC21 & $0 / 5$ & $0 / 5$ & $0 / 5$ & $0 / 5$ & $0 / 5$ & $0 / 5$ & $0 / 5$ & $1 / 5$ & $0 / 5$ & $0 / 5$ & $0 / 5$ & $0 / 5$ \\
\hline & & CC26 & $0 / 3$ & $0 / 3$ & $0 / 3$ & $0 / 3$ & $0 / 3$ & $0 / 3$ & $0 / 3$ & $0 / 3$ & $0 / 3$ & $0 / 3$ & $0 / 3$ & $0 / 3$ \\
\hline & & $\mathrm{CC} 29$ & $0 / 3$ & $0 / 3$ & $0 / 3$ & $0 / 3$ & $0 / 3$ & $0 / 3$ & $0 / 3$ & $0 / 3$ & $0 / 3$ & $0 / 3$ & $0 / 3$ & $0 / 3$ \\
\hline & & CC31 & 0/1 & $0 / 1$ & 0/1 & 0/1 & $0 / 1$ & $0 / 1$ & $0 / 1$ & $1 / 1$ & $0 / 1$ & $0 / 1$ & 0/1 & $0 / 1$ \\
\hline & & CC37 & $0 / 9$ & 0/9 & $0 / 9$ & $0 / 9$ & $0 / 9$ & $0 / 9$ & $0 / 9$ & $0 / 9$ & $0 / 9$ & $0 / 9$ & $0 / 9$ & $0 / 9$ \\
\hline & & CC101 & $1 / 4$ & $0 / 4$ & $0 / 4$ & $0 / 4$ & $0 / 4$ & $0 / 4$ & $0 / 4$ & $0 / 4$ & $0 / 4$ & $0 / 4$ & $0 / 4$ & $0 / 4$ \\
\hline & & CC121 & $9 / 14$ & $0 / 14$ & $0 / 14$ & $0 / 14$ & $0 / 14$ & $0 / 14$ & $0 / 14$ & $13 / 14$ & $0 / 14$ & $0 / 14$ & $0 / 14$ & $0 / 14$ \\
\hline & & CC124 & $0 / 2$ & $0 / 2$ & $0 / 2$ & $0 / 2$ & $0 / 2$ & $0 / 2$ & $0 / 2$ & $2 / 2$ & $0 / 2$ & $0 / 2$ & $0 / 2$ & $0 / 2$ \\
\hline & & CC155 & $0 / 8$ & $0 / 8$ & $0 / 8$ & $0 / 8$ & $0 / 8$ & $0 / 8$ & $0 / 8$ & $1 / 8$ & $0 / 8$ & $0 / 8$ & $0 / 8$ & $0 / 8$ \\
\hline & & CC193 & $0 / 1$ & $0 / 1$ & $0 / 1$ & $0 / 1$ & $0 / 1$ & $0 / 1$ & $0 / 1$ & $1 / 1$ & $0 / 1$ & $0 / 1$ & $0 / 1$ & $0 / 1$ \\
\hline & & $\mathrm{CC} 200$ & $0 / 1$ & $0 / 1$ & $0 / 1$ & $0 / 1$ & $0 / 1$ & $0 / 1$ & $0 / 1$ & $0 / 1$ & $0 / 1$ & $0 / 1$ & $0 / 1$ & $0 / 1$ \\
\hline & & CC204 & $0 / 6$ & $0 / 6$ & $0 / 6$ & $0 / 6$ & $0 / 6$ & $2 / 6$ & $2 / 6$ & $0 / 6$ & $0 / 6$ & $6 / 6$ & $6 / 6$ & $0 / 6$ \\
\hline & & CC398 & 0/1 & $0 / 1$ & 0/1 & $0 / 1$ & $0 / 1$ & $0 / 1$ & $0 / 1$ & $0 / 1$ & $0 / 1$ & $0 / 1$ & $0 / 1$ & $0 / 1$ \\
\hline & & $\mathrm{CC} 403$ & $0 / 3$ & $0 / 3$ & $0 / 3$ & $0 / 3$ & $0 / 3$ & $0 / 3$ & $0 / 3$ & $0 / 3$ & $0 / 3$ & $0 / 3$ & $0 / 3$ & $0 / 3$ \\
\hline & & CC415 & 0/1 & $0 / 1$ & $0 / 1$ & $0 / 1$ & $0 / 1$ & $0 / 1$ & $0 / 1$ & $0 / 1$ & $0 / 1$ & $0 / 1$ & $0 / 1$ & $0 / 1$ \\
\hline & & CC451 & $0 / 16$ & $0 / 16$ & $0 / 16$ & $0 / 16$ & $0 / 16$ & $0 / 16$ & $0 / 16$ & $0 / 16$ & $0 / 16$ & $0 / 16$ & $0 / 16$ & $0 / 16$ \\
\hline & & CC475 & $0 / 3$ & $0 / 3$ & $0 / 3$ & $0 / 3$ & $0 / 3$ & $0 / 3$ & $0 / 3$ & $0 / 3$ & $0 / 3$ & $0 / 3$ & $0 / 3$ & $0 / 3$ \\
\hline & $1 / 2 \mathrm{c}$ & CC9 & $1 / 15$ & $0 / 15$ & $0 / 15$ & $0 / 15$ & 0/15 & $0 / 15$ & $0 / 15$ & $5 / 15$ & $0 / 15$ & $0 / 15$ & $0 / 15$ & $15 / 15$ \\
\hline
\end{tabular}

FIGURE 1 | Heatmaps show the association of the occurrence of benzalkonium chloride, cadmium, and arsenic resistance genes (A) with L. monocytogenes origin and (B) with clonal complexes. CCs, clonal complexes. In columns for individual genes, the number of positive strains/number of tested strains of the specific CC, the intensity of blue color expresses the percentage.

(27.8\%) and arsenic (15.6\%) in L. monocytogenes strains isolated from humans, food, and the environment in the Czechia correlates with the high prevalence of heavy metal resistance described in studies from other countries (McLauchlin et al., 1997; Mullapudi et al., 2008; Xu et al., 2014; Lee et al., 2017). The higher proportion of cadmium resistance genes in comparison with arsenic resistance genes could be explained by a more frequent environmental exposure of L. monocytogenes to cadmium. L. monocytogenes can be commonly isolated from the environment of farm animals. Zhen et al. (2020) reported in their study that continuous application of manure over 15 years has significantly increased the concentration of cadmium in the soil (by 230\%), while the concentration of arsenic was relatively stable.

Regarding cadmium resistance genes, mainly cadA1C1 genes, predominated (17.8\% of strains), and conversely, chromosomally located cadA3C3 genes were not detected in any strain, which is in accordance with other studies (Ratani et al., 2012; Lee et al., 2013; $\mathrm{Xu}$ et al., 2014; Zhang et al., 2015). Some studies reported that all benzalkonium chloride-resistant strains were also cadmiumresistant (Mullapudi et al., 2008; Ratani et al., 2012; Zhang et al., 2015). However, this coresistance cannot always be explained by the presence of the $b c r A B C$ cassette and $c a d A 2 C 2$, which are carried on the same plasmid. In our study, the co-occurrence of genetic determinants encoding resistance to cadmium and benzalkonium chloride was demonstrated in $4.8 \%$ strains and the plasmid carrying $b c r A B C$ cassette and cadA2C2 operon in only $1.1 \%$ of strains isolated from different sources. In some strains, the phenotypic manifestation of resistance or tolerance to benzalkonium chloride could also be affected by the multidrug chromosomal efflux pump MdrL (Jiang et al., 2019), which was detected in the genome of all tested strains. 
Benzalkonium chloride resistance genes in L. monocytogenes were detected in only $7 \%$ of the strains tested in this study compared to cadmium and arsenic resistance genes. The analysis of almost 3000 L. monocytogenes genomes in France has shown that some of the benzalkonium chloride resistance genes have been identified in $32.1 \%$ of strains obtained by surveillance of listeriosis (Maury et al., 2019). A high prevalence of benzalkonium chloride resistance in L. monocytogenes was also found in another study characterizing 100 L. monocytogenes strains from three meat and vegetable food-processing plants, with the occurrence of resistance genes in $62 \%$ strains, increasing to $73 \%$ among suspect persistent strains (Hurley et al., 2019). In the collection of $L$. monocytogenes from Switzerland and Finland, resistance to benzalkonium chloride was demonstrated in 12.3 and $10.6 \%$ of strains isolated mainly from food, but also from humans and wild bird feces (Meier et al., 2017). The lower prevalence of benzalkonium chloride resistance genes in L. monocytogenes strains isolated in the Czechia might have been caused by selection of the tested strains in different studies, but also by habits in using quaternary ammonium compounds as disinfection agents in food-processing plants in particular countries.

Regarding benzalkonium chloride resistance, Maury et al. (2019) in France most frequently detected the qacH gene (18.8\%), followed by the bcrABC (8.2\%), emrC (5\%), qacC (0.1\%), emrE $(0.03 \%)$, and qacA $(0.03 \%)$ genes. As part of the LiSEQ project (Listeria SEQuencing), in a collection of strains from the European Union derived from ready-to-eat foods, food animals, food-processing environments, and humans, the qacH gene was detected in almost the same proportions (in 18.5\% of strains), similarly to $b c r A B C$ ( $5 \%$ strains) and the $e m r E$ and qacA genes ( $<1 \%$ of strains) (Painset et al., 2019). In L. monocytogenes strains originating in Switzerland and Finland, resistance to benzalkonium chloride was also most frequently encoded by the qacH gene, namely, in approximately $57 \%$ of strains (Meier et al., 2017). Our findings are consistent with the above study as in our study the $q a c H$ gene predominated in the tested group, despite the lower prevalence of benzalkonium chloride resistance genes in strains isolated from humans, food, and the environment in the Czechia (5.6\%).

\section{Occurrence of Genetic Determinants Encoding Cadmium, Arsenic, and Benzalkonium Chloride Resistance Depending on Species Heterogeneity of L. monocytogenes Strains}

The genes encoding cadmium, arsenic, and benzalkonium chloride resistance in the tested strains of $L$. monocytogenes were carried mainly on mobile genetic elements. The detection of specific resistance genes in phylogenetically distant lineages I and II is indicative of their potential horizontal spread (Table 2). However, the ability to resist or tolerate heavy metals and disinfectants could provide an advantage to certain L. monocytogenes serotypes and CCs in their spread. The results showed that rather than the source of $L$. monocytogenes, the occurrence of resistance genes to cadmium, arsenic, and benzalkonium chloride is associated with certain serotypes and CCs, the distribution of which may differ in various countries. A number of studies have reported a frequent occurrence of cadmium resistance genes and a sporadic occurrence of arsenic resistance genes in serotype $1 / 2$ a strains in comparison with serotype 4b strains (McLauchlin et al., 1997; Mullapudi et al., 2008; Lee et al., 2017). In the present study, the occurrence of cadmium resistance genes was similar in the strains of all serotypes tested: $1 / 2 \mathrm{a}(27.9 \%, 38 / 136), 1 / 2 \mathrm{c}(33.3 \%, 5 / 15), 4 \mathrm{~b}$ $(25.3 \%, 20 / 79)$, and $1 / 2 \mathrm{~b}(32.5 \%, 13 / 40)$. In contrast, arsenic resistance genes occurred only rarely in serotype $1 / 2$ a strains $(5.1 \%, 7 / 136)$ and were not detected in any strain of serotype $1 / 2 \mathrm{~b}$, which is in accordance with other studies (McLauchlin et al., 1997; Mullapudi et al., 2008; Ratani et al., 2012), despite the fact that they belong to the same evolutionary lineage as strains of serotype $4 \mathrm{~b}$. In this study, arsenic resistance genes were detected more frequently in serotype $1 / 2 \mathrm{c}$ strains $(100 \%, 15 / 15)$ than in serotype $4 \mathrm{~b}$ strains $(25.3 \%, 20 / 79)$.

In the Czechia, L. monocytogenes serotype $1 / 2 \mathrm{a}$, with frequent occurrence of CC8, has long prevailed not only in humans, but also in foods (Tomáštíková et al., 2019). In CC8 strains tested in the current study obtained from both clinical cases of listeriosis $(n=18)$ and food $(n=13)$, we demonstrated a relatively frequent occurrence $(35.5 \%)$ of cadmium resistance genes cadA1C1 located on the transposon Tn5422 related to plasmid. On the other hand, in many European countries, strains of serotype $4 \mathrm{~b}$ of CCs CC1, CC2, CC4, and CC6 predominated in clinical cases of listeriosis (Painset et al., 2019). In the genome of strains CC2 (84\%), CC1 (35\%), and CC4 (24\%), but not in strain CC6, Lee et al. (2017) demonstrated the presence of LGI2 in which cadmium and arsenic resistance genes are located simultaneously. They reported that LGI2 shows significant content plasticity and is capable of transferring different accessory genes to diverse chromosomal locations. In our study, we demonstrated LGI2 mainly in the CC2 (20/21), associated with hypervirulence (Maury et al., 2019), but not in CC1 and CC4 strains. However, the effect of LGI2 on L. monocytogenes virulence has not yet been elucidated. For example, in the Czechia, within serotype 4b, CC6 strains predominate among clinical cases of listeriosis (Tomáštíková et al., 2019), in which this genetic island does not occur.

While in strains of serotypes $4 b$ and $1 / 2 a$, the arsenic resistance genes were located on LGI2, all tested 1/2c strains of CC9 carried in the genome arsenic resistance genes on Tn554 located on the chromosome. Our results are consistent with previous studies describing more frequent occurrence of arsenic resistance genes on Tn554 in L. monocytogenes serotypes $1 / 2 \mathrm{c}$ and $1 / 2$ a (Kuenne et al., 2013; Lee et al., 2017). With the exception of one human strain $1 / 2 c$, the other strains of serotype $1 / 2 c$ tested in this study $(n=14)$ were derived from food sources. Within serotype 1/2c, McLauchlin et al. (1997) reported more frequent cadmium resistance and less frequent arsenic resistance in strains derived from clinical cases of listeriosis in comparison with food strains. This could explain the high occurrence of arsenic resistance genes found in strains $1 / 2 c$ tested in this study. In the Czechia, L. monocytogenes serotype $1 / 2 \mathrm{c}$ is very rarely associated with 
human listeriosis cases (Tomáštíková et al., 2019), and therefore, it was not possible to compare these findings.

It was noteworthy that all CC204 strains tested in this study carried LGI2 regardless of their origin. The tested group of strains CC204 $(n=6)$ included strains isolated from the environment, vegetables, and persistent strains from two cheese-processing plants (Supplementary Table). The occurrence of LGI2 has already been detected in CC204 strains (Hingston et al., 2019; Palma et al., 2020), as well as in strains CC14 and CC121 (Pasquali et al., 2018). However, in this study, LGI2 was detected in only one of the five CC14 strains isolated from fresh cheese and in none of the CC121 strains. In France, LGI2 has also been detected in persistent strains of L. monocytogenes CC204 derived from seafood-processing plants (Palma et al., 2020). Even though the role of LGI2 in L. monocytogenes persistence is not clear, current studies suggested that mobile genetic elements could improve Listeria survival rates in the environment of food-processing plants (Pirone-Davies et al., 2018; Palma et al., 2020).

In addition to cadmium resistance, more frequent resistance to benzalkonium chloride has been reported in lineage II strains (serotypes $1 / 2 \mathrm{a}$ and $1 / 2 \mathrm{c}$ ) in a number of European studies (Meier et al., 2017; Pirone-Davies et al., 2018; Maury et al., 2019; Painset et al., 2019) and in the United States (Mullapudi et al., 2008; Ratani et al., 2012). In our study, benzalkonium chloride resistance genes were also detected more frequently in lineage II strains. On the other hand, in a Chinese study, benzalkonium chloride resistance was detected mainly in L. monocytogenes serotype $4 \mathrm{~b}$ isolated from food, probably due to a very low number of resistant strains tested ( $\mathrm{Xu}$ et al., 2014). The results of a number of studies suggested that the occurrence of benzalkonium chloride resistance genes is mainly associated with CC9 and CC121 (Meier et al., 2017; Maury et al., 2019). This is confirmed by the results of our study, where more than half of the tested CC121 strains $(60 \%, 9 / 15)$ carried the qacH gene. In L. monocytogenes CC9, in which arsenic resistance predominated, benzalkonium chloride resistance genes were detected in $7 \%(1 / 15)$ of the strains tested. For strains of the CC121 CC, the results of this and other studies (Maury et al., 2019) also suggested coresistance to cadmium. With the exception of one strain isolated from a meat product, all L. monocytogenes CC121 tested in this study isolated from food, humans, and the environment carried cadA1C1_Tn5422 (Supplementary Table).

Because of the fact that the majority of cadmium, arsenic, and benzalkonium chloride resistance genes are carried on mobile genetic elements with the possibility of horizontal spread, the occurrence of these genes is not always related to all strains of a particular L. monocytogenes CC, as shown by the results of the present study (Figures 1A,B). An example is the occurrence of the Emrc efflux pump carried on plasmid pLMST6, originally described for strains of serotype 4b CC6 (Kremer et al., 2017), and also for strains of serotype 1/2a CC8, CC31, and CC403 isolated from humans and food (Kropac et al., 2019). In contrast, in our study, a plasmid carrying the emrC gene was detected in a single strain of CC8 $(0.4 \%)$ and in none of the tested strains CC31 and CC403 isolated from human listeriosis cases, food, and the environment.

\section{CONCLUSION}

In the present study, no differences were found in the occurrence and type of genetic determinants encoding resistance to heavy metals such as cadmium and arsenic and the disinfecting agent benzalkonium chloride between strains isolated from human listeriosis cases and food. The higher prevalence of resistance genes, especially to cadmium, but also to arsenic, compared to benzalkonium chloride resistance genes may indicate a higher selection pressure of the environment due to heavy metal contamination and limited use of benzalkonium chloride for disinfection in the Czechia. The distribution of cadmium, arsenic, and benzalkonium chloride resistance genes carried mainly on mobile genetic elements showed affinity for specific CCs, regardless of the source of L. monocytogenes. This may be the reason for sporadic finding of heavy metal resistance genes in $L$. monocytogenes strains originating from the environment of the Czechia tested in this study. The obtained results point to the need for further research into the characteristics of L. monocytogenes isolated from environmental sources to understand the dissemination of specific clones throughout the food chain.

\section{DATA AVAILABILITY STATEMENT}

The datasets presented in this study can be found in online repositories. The names of the repository/repositories and accession number(s) can be found below: the European Nucleotide Archive (ENA) at EMBL-EBI under accession number PRJEB37840 (https://www. ebi.ac.uk/ena/data/view/PRJEB37840) and PRJEB40064 (https://www.ebi.ac.uk/ena/data/view/PRJEB40064).

\section{AUTHOR CONTRIBUTIONS}

All the authors read and agreed to the published version of the manuscript. TG and RK performed the concept and methodology of this study. TG wrote the manuscript. KK and NS extracted genomic DNA for WGS. MF, $\mathrm{LH}$, and AK performed the bio-informatic analysis. All authors contributed to the article and approved the submitted version.

\section{FUNDING}

This study was supported by grant of the Ministry of Education, Youth and Sport CZ.1.05./2.1.00/19.0385, Ministry of Agriculture QK1910121, and the European Joint Programme One Health, European Union's Horizon 2020 Research and Innovation Program, under Grant Agreement No. 773830.

\section{SUPPLEMENTARY MATERIAL}

The Supplementary Material for this article can be found online at: https://www.frontiersin.org/articles/10.3389/fmicb.2020. 599882/full\#supplementary-material 


\section{REFERENCES}

Carattoli, A., Zankari, E., García-Fernández, A., Voldby Larsen, M., Lund, O., Villa, L., et al. (2014). In silico detection and typing of plasmids using PlasmidFinder and plasmid multilocus sequence typing. Antimicrob. Agents Chemother. 58, 3895-3903. doi: 10.1128/AAC.02412-14

Carpentier, B., and Cerf, O. (2011). Review-Persistence of Listeria monocytogenes in food industry equipment and premises. Int. J. Food Microbiol. 145, 1-8. doi: 10.1016/j.ijfoodmicro.2011.01.005

Doležalová Weissmannová, H., and Pavlovský, J. (2017). Indices of soil contamination by heavy metals - methodology of calculation for pollution assessment (minireview). Environ. Monit. Assess. 189:616. doi: 10.1007/s10661017-6340-5

Elhanafi, D., Dutta, V., and Kathariou, S. (2010). Genetic characterization of plasmid-associated benzalkonium chloride resistance determinants in a Listeria monocytogenes strain from the 1998-1999 outbreak. Appl. Environ. Microbiol. 76, 8231-8238. doi: 10.1128/AEM.02056-10

Gray, M. J., Freitag, N. E., and Boor, K. J. (2006). How the bacterial pathogen Listeria monocytogenes mediates the switch from environmental Dr. Jekyll to pathogenic Mr. Hyde. Infect. Immun. 74, 2505-2512. doi: 10.1128/IAI.74.5. 2505-2512.2006

Harvey, J., and Gilmour, A. (2001). Characterization of recurrent and sporadic Listeria monocytogenes isolates from raw milk and nondairy foods by pulsedfield gel electrophoresis, monocin typing, plasmid profiling, and cadmium and antibiotic resistance determination. Appl. Environ. Microbiol. 67, 840-847. doi: 10.1128/AEM.67.2.840-847.2001

Hingston, P., Brenner, T., Hansen, L. T., and Wang, S. (2019). Comparative analysis of Listeria monocytogenes plasmids and expression levels of plasmid-encoded genes during growth under salt and acid stress conditions. Toxins 11:426. doi: $10.3390 /$ toxins 11070426

Hurley, D., Luque-Sastre, L., Parker, C. T., Huynh, S., Eshwar, A. K., Nguyen, S. V., et al. (2019). Whole-genome sequencing-based characterization of 100 Listeria monocytogenes isolates collected from food processing environments over a four-year period. mSphere 4:e00252-19. doi: 10.1128/mSphere.00252-19

Jiang, X., Yu, T., Xu, Y., Wang, H., Korkeala, H., and Shi, L. (2019). MdrL, a major facilitator superfamily efflux pump of Listeria monocytogenes involved in tolerance to benzalkonium chloride. Appl. Microbiol. Biotechnol. 103, 13391350. doi: 10.1007/s00253-018-9551-y

Katharios-Lanwermeyer, S., Rakic-Martinez, M., Elhanafi, D., Ratani, S., Tiedje, J. M., and Kathariou, S. (2012). Coselection of cadmium and benzalkonium chloride resistance in conjugative transfers from nonpathogenic Listeria spp. to other Listeriae. Appl. Environ. Microbiol. 78, 7549-7556. doi: 10.1128/AEM. 02245- 12

Korsak, D., Chmielowska, C., Szuplewska, M., and Bartosik, D. (2019). Prevalence of plasmid-borne benzalkonium chloride resistance cassette $\operatorname{bcr} A B C$ and cadmium resistance cadA genes in nonpathogenic Listeria spp. isolated from food and food-processing environments. Int. J. Food Microbiol. 290, 247-253. doi: 10.1016/j.ijfoodmicro.2018.10.019

Koudelka, Š, Gelbíčová, T., Procházková, M., and Karpíšková, R. (2018). Lineage and serotype identification of Listeria monocytogenes by matrix-assisted laser desorption ionization-time of flight mass spectrometry. Czech J. Food Sci. 36, 452-458. doi: 10.17221/87/2018-CJFS

Kovacevic, J., Ziegler, J., Wałecka-Zacharska, E., Reimer, A., Kitts, D. D., and Gilmour, M. W. (2015). Tolerance of Listeria monocytogenes to quaternary ammonium sanitizers is mediated by a novel efflux pump encoded by emrE. Appl. Environ. Microbiol. 82, 939-953. doi: 10.1128/AEM.03741-15

Kremer, P. H. C., Lees, J. A., Koopmans, M. M., Ferwerda, B., Arends, A. W. M., Feller, M. M., et al. (2017). Benzalkonium tolerance genes and outcome in Listeria monocytogenes meningitis. Clin. Microbiol. Infect. 23, 265.e1-265.e7. doi: 10.1016/j.cmi.2016.12.008

Kropac, A. C., Eshwar, A. K., Stephan, R., and Tasara, T. (2019). New insights on the role of the pLMST6 plasmid in Listeria monocytogenes biocide tolerance and virulence. Front. Microbiol. 10:1538. doi: 10.3389/fmicb.2019.01538

Kuenne, C., Billion, A., Mraheil, M. A., Strittmatter, A., Daniel, R., Goesmann, A., et al. (2013). Reassessment of the Listeria monocytogenes pan-genome reveals dynamic integration hotspots and mobile genetic elements as major components of the accessory genome. BMC Genomics 14:47. doi: 10.1186/14712164-14- 47
Lee, S., Rakic-Martinez, M., Graves, L. M., Ward, T. J., Siletzky, R. M., and Kathariou, S. (2013). Genetic determinants for cadmium and arsenic resistance among Listeria monocytogenes serotype $4 \mathrm{~b}$ isolates from sporadic human listeriosis patients. Appl. Environ. Microbiol. 79, 2471-2476. doi: 10.1128/AEM. 03551- 12

Lee, S., Ward, T. J., Jima, D. D., Parsons, C., and Kathariou, S. (2017). The arsenic resistance-associated Listeria genomic island LGI2 exhibits sequence and integration site diversity and a propensity for three Listeria monocytogenes clones with enhanced virulence. Appl. Environ. Microbiol. 83:e01189-17. doi: 10.1128/AEM.01189-17

Maury, M. M., Bracq-Dieye, H., Huang, L., Vales, G., Lavina, M., Thouvenot, P., et al. (2019). Hypervirulent Listeria monocytogenes clones' adaption to mammalian gut accounts for their association with dairy products. Nat. Commun. 10:2488. doi: 10.1038/s41467-019-10380-0

McLauchlin, J., Hampton, M. D., Shah, S., Threlfall, E. J., Wieneke, A. A., and Curtis, G. D. W. (1997). Subtyping of Listeria monocytogenes on the basis of plasmid profiles and arsenic and cadmium susceptibility. J. Appl. Microbiol. 83, 381-388. doi: 10.1046/j.1365-2672.1997.00238.x

Meier, A. B., Guldimann, C., Markkula, A., Pöntinen, A., Korkeala, H., and Tasara, T. (2017). Comparative phenotypic and genotypic analysis of Swiss and Finnish Listeria monocytogenes isolates with respect to benzalkonium chloride resistance. Front. Microbiol. 8:397. doi: 10.3389/fmicb.2017.00397

Mullapudi, S., Siletzky, R. M., and Kathariou, S. (2008). Heavy-metal and benzalkonium chloride resistance of Listeria monocytogenes isolates from the environment of turkey-processing plants. Appl. Environ. Microbiol. 74, 14641468. doi: 10.1128/AEM.02426-07

Müller, A., Rychli, K., Muhterem-Uyar, M., Zaiser, A., Stessl, B., Guinane, C. M., et al. (2013). Tn6188 - a novel transposon in Listeria monocytogenes responsible for tolerance to benzalkonium chloride. PLoS One 8:e76835. doi: 10.1371/ journal.pone.0076835

Okonechnikov, K., Golosova, O., Fursov, M., and The UGENE Team (2012). Unipro UGENE: a unified bioinformatics toolkit. Bioinformatics 28, 1166-1167. doi: 10.1093/bioinformatics/bts091

Painset, A., Björkman, J. T., Kiil, K., Guillier, L., Mariet, J. F., Félix, B., et al. (2019). LiSEQ - whole-genome sequencing of a cross-sectional survey of Listeria monocytogenes in ready-to-eat foods and human clinical cases in Europe. Microb. Genom. 5:e000257. doi: 10.1099/mgen.0.000257

Palma, F., Brauge, T., Radomski, N., Mallet, L., Felten, A., Mistou, M. Y., et al. (2020). Dynamics of mobile genetic elements of Listeria monocytogenes persisting in ready-to-eat seafood processing plants in France. BMC Genomics 21:130. doi: 10.1186/s12864-020-6544-x

Parsons, C., Lee, S., and Kathariou, S. (2019). Heavy metal resistance determinants of the foodborne pathogen Listeria monocytogenes. Genes. 10:11. doi: 10.3390/ genes 10010011

Parsons, C., Lee, S., and Kathariou, S. (2020). Dissemination and conservation of cadmium and arsenic resistance determinants in Listeria and other grampositive bacteria. Mol. Microbiol. 113, 560-569. doi: 10.1111/mmi.14470

Pasquali, F., Palma, F., Guillier, L., Lucchi, A., De Cesare, A., and Manfreda, G. (2018). Listeria monocytogenes sequence types 121 and 14 repeatedly isolated within one year of sampling in a rabbit meat processing plant: persistence and ecophysiology. Front. Microbiol. 9:596. doi: 10.3389/fmicb.2018.00596

Pirone-Davies, C., Chen, Y., Pightling, A., Ryan, G., Wang, Y., Yao, K., et al. (2018). Genes significantly associated with lineage II food isolates of Listeria monocytogenes. BMC Genomics 19:708. doi: 10.1186/s12864-018-5074-2

Pombinho, R., Camejo, A., Vieira, A., Reis, O., Carvalho, F., Almeida, M. T., et al. (2017). Listeria monocytogenes CadC regulates cadmium efflux and finetunes lipoprotein localization to escape the host immune response and promote infection. J. Infect. Dis. 215, 1468-1479. doi: 10.1093/infdis/jix118

Ratani, S. S., Siletzky, R. M., Dutta, V., Yildirim, S., Osborne, J. A., Lin, W., et al. (2012). Heavy metal and disinfectant resistance of Listeria monocytogenes from foods and food processing plants. Appl. Environ. Microbiol. 78, 6938-6945. doi: 10.1128/AEM.01553-12

Tomáštíková, Z., Gelbíčová, T., and Karpíšková, R. (2019). Population structure of Listeria monocytogenes isolated from human listeriosis cases and from ready-to-eat foods in the Czech Republic. J. Food Nut. Res. 58, 99-106.

Xu, D., Li, Y., Zahid, M. S. H., Yamasaki, S., Shi, L., Li, J. R., et al. (2014). Benzalkonium chloride and heavy-metal tolerance in Listeria monocytogenes 
from retail foods. Int. J. Food Microbiol. 190, 24-30. doi: 10.1016/j.ijfoodmicro. 2014.08.017

Zhang, H., Zhou, Y., Bao, H., Zhang, L., Wang, R., and Zhou, X. (2015). Plasmid-borne cadmium resistant determinants are associated with the susceptibility of Listeria monocytogenes to bacteriophage. Microbiol. Res. 172, 1-6. doi: 10.1016/j.micres.2015. 01.008

Zhen, H., Jia, L., Huang, C., Qiao, Y., Li, J., Li, H., et al. (2020). Long-term effects of intensive application of manure on heavy metal pollution risk in protected-field vegetable production. Environ. Pollut. 263:114552. doi: 10.1016/j.envpol.2020. 114552
Conflict of Interest: The authors declare that the research was conducted in the absence of any commercial or financial relationships that could be construed as a potential conflict of interest.

Copyright (C) 2021 Gelbicova, Florianova, Hluchanova, Kalova, Korena, Strakova and Karpiskova. This is an open-access article distributed under the terms of the Creative Commons Attribution License (CC BY). The use, distribution or reproduction in other forums is permitted, provided the original author(s) and the copyright owner(s) are credited and that the original publication in this journal is cited, in accordance with accepted academic practice. No use, distribution or reproduction is permitted which does not comply with these terms. 